\title{
Carbohydrate metabolism by murine ovarian follicles and oocytes grown in vitro
}

\author{
Sarah E Harris, Iris Adriaens ${ }^{1}$, Henry J Leese ${ }^{2}$, Roger G Gosden ${ }^{3}$ and Helen M Picton \\ Reproduction and Early Development Research Group, Leeds Institute of Genetics, Health and Therapeutics, \\ University of Leeds, Leeds LS2 9JT, UK, ${ }^{1}$ Follicle Biology Laboratory, Center for Reproductive Medicine, Free \\ University Brussels, Laarbeeklaan 101, 1090 Brussels, Belgium, ${ }^{2}$ Department of Biology, University of York, PO Box \\ 373, York YO10 5YW, UK and ${ }^{3}$ Center for Reproductive Medicine and Infertility, Weill Medical College of Cornell \\ University, New York, New York 10021, USA
}

Correspondence should be addressed to S E Harris; Email: s.e.harris@leeds.ac.uk

\begin{abstract}
Metabolic markers are potentially valuable for assessment of follicle development in vitro. Carbohydrate metabolism of murine preantral follicles grown to maturity over 13 days in vitro has been measured, and metabolism of resulting oocyte-cumulus complexes (OCCs) and denuded oocytes has been compared with in vivo ovulated control counterparts. Spent follicle culture media were analysed for glucose, lactate and pyruvate concentrations. During follicle in vitro growth, glycolysis accounted for a rise from $\sim 24$ to $60 \%$ of all glucose consumed. Ovulation induction caused a significant increase in glucose uptake and lactate production by in vitrogrown follicles to $71.7 \pm \mathbf{1 . 2}$ and $\mathbf{9 6 . 6} \pm \mathbf{4 . 8} \mathbf{n m o l e s} /$ day respectively. OCCs grown in vitro had significantly higher rates of glucose consumption and lactate and pyruvate production $(110.1 \pm 3.5,191.8 \pm 8.9$ and $31.7 \pm 1.7 \mathrm{pmoles} / \mathrm{h}$ respectively) than in vivo ovulated controls $(67.4 \pm 8.1,113.9 \pm 17.1$ and $20.2 \pm 4.0 \mathrm{pmoles} / \mathrm{h}$ respectively), but a reduced capacity for pyruvate consumption $(1.13 \pm 0.06$ vs $1.49 \pm 0.06$ pmoles/h by in vivo ovulated oocytes). Metabolism of OCCs was affected by the quality of the original follicle. In vitro-grown oocytes had a reduced cytoplasmic volume when compared with controls (168.3 \pm 2.0 vs $199.0 \pm 3.2$ proportionately respectively) but a similar rate of metabolism per unit volume. Meiotic status influenced metabolism of both OCCs and denuded oocytes. In conclusion, glucose consumption and lactate production by cultured follicles increased in tandem with developmental progression and were stimulated prior to ovulation. Additionally, the metabolic profiles of in vitro produced OCCs and the oocytes within them are affected by long-term exposure to the culture environment.
\end{abstract}

Reproduction (2007) 134 415-424

\section{Introduction}

Improved understanding of the physiology of mammalian ovarian follicle growth and how this is affected by long-term culture is critical to the development of technologies for preservation of fertility. Moreover, long-term culture of ovarian follicles may become an important strategy for the production of oocytes in vitro (Picton et al. 2003). The potential value of this procedure is widely recognised; however, the technology remains imperfect, hindered by extremely demanding methodologies and incomplete understanding of the biology of oogenesis and folliculogenesis in vivo and in vitro. Depending upon species, it may take many weeks or months for a primordial follicle to undergo growth initiation and development to support the production of a fully grown fertile oocyte. The process of folliculogenesis involves many intricate and timely developmental events, which must be replicated in the laboratory.
Avascular preantral follicles survive relatively well in vitro and culture of preantral follicles is possible in several species, including mouse (Nayudu \& Osborn 1992, Boland et al. 1993, Cortvrindt et al. 1996, Rose etal. 1999), sheep (Newton et al. 1999, Picton et al. 2003) and human (Roy \& Treacy 1993, Picton \& Gosden 2000). However, after long-term culture, oocyte viability is reduced and this is especially a problem in large animals and humans. Culture conditions are probably suboptimal in all species, resulting in low yields of oocytes potentially causing epigenetic alterations and imprinting defects as well as compromised cytoplasmic maturation and reduced developmental competence (Obata et al. 2002, Fernandez-Gonzalez et al. 2004, Calder et al. 2005).

Culture conditions required to improve follicle and oocyte growth in vitro must be re-evaluated based, in part, on the energy and nutritional requirements of follicles and oocytes grown in vivo. Two carbohydrates 
stand out with obvious importance: glucose is a major energy substrate for mouse ovarian follicles (Boland et al. 1993, 1994a, 1994b), whereas pyruvate is essential for the mouse oocyte (Biggers et al. 1967, Harris 2002).

Glucose metabolism by the late preantral and antral mammalian ovarian follicle is thought to be predominantly glycolytic (Leese \& Lenton 1990, Boland et al. 1993, 1994a, 1994b, Gull etal. 1999), thus reducing the demand for oxygen which would otherwise be exhausted within the outer follicle layers (Gosden \& Byatt-Smith 1986). This strategy presumably conserves oxygen for use by the oocyte, whose ATP production is confined to catabolism of nutrients, such as pyruvate (Biggers et al. 1967), through oxidative metabolism. Oxygen is an absolute requirement for energy metabolism by the oocyte and, hence, hypoxia in antral follicles is expected to reduce oocyte developmental competence (Van Blerkom 1998).

While glycolysis is an important pathway for glucose utilisation by larger follicles, its contribution to carbohydrate metabolism by smaller follicles has not been reported. Preantral follicles are less likely to suffer from the limitations of diffusion because they are $<0.3-0.4 \mathrm{~mm}$ in diameter (Gosden \& Byatt-Smith 1986), and could feasibly utilise other, more efficient, pathways for energy production. In this study, we have used the mouse as a model to study carbohydrate metabolism in mammalian preantral follicles. We describe glucose, lactate and pyruvate metabolism by murine follicles grown in vitro over 13 days to maturity from the late primary/early secondary stage. Nutrient metabolism of in vitro-grown oocytecumulus complexes (OCCs) and ovulated oocytes derived from them has been compared with stage-matched controls grown in vivo to reveal the physiological impact of the culture environment.

\section{Materials and Methods}

Unless otherwise stated, all chemicals were purchased from Sigma Chemical Company.

\section{Animals}

Female $\mathrm{F} 1$ mice were derived from $\mathrm{C} 57 / \mathrm{Bl} 6 \times \mathrm{CBA} / \mathrm{Ca} / \mathrm{Br} /$ matings. They were housed in controlled conditions, subjected to $13 \mathrm{~h}$ light: $11 \mathrm{~h}$ darkness with water and food available ad libitum. Mice were killed using a schedule one procedure of $\mathrm{CO}_{2}$ asphyxiation, followed by cervical dislocation. Animal husbandry and experimental procedures were in accordance with licences held by RGG and HJL from the Home Office of the United Kingdom.

\section{Follicle isolation and culture}

Preantral follicles of $100-130 \mu \mathrm{m}$ diameter at the late primary/early secondary transition were isolated from the ovaries of 14- to 16-day-old mice under a stereomicroscope using two sterile 27-gauge needles. Follicle diameter was recorded and the degree of theca coverage (Cortvrindt et al. 1996) was graded as either grade $1(>50 \%)$ or $2(<50 \%)$. The apposition between the oocyte and the surrounding granulosa cells was also noted (Cortvrindt et al. 1996) and was characterised as the degree of close contact or adhesion between an oocyte and its immediate granulosa neighbours. Follicles with very little or no discernable disruption or gap between oocyte and granulosa cells were classified as grade 1 ( $<1 / 3$ disrupted oocyte-granulosa cell contact), follicles in which a gap could be seen affecting $1 / 3-2 / 3$ of granulosa cells immediately surrounding the oocyte were classified as grade 2, whereas grade 3 follicles showed poor cell-cell contact between oocyte and corona, with oocyte-granulosa disruption affecting $>2 / 3$ of corona cells. Follicles were cultured using the method of Cortvrindt et al. (1996) in $20 \mu \mathrm{l}$ droplets of $\alpha$-MEM supplemented with ITS (5 $\mu \mathrm{g} /$ $\mathrm{ml}$ insulin, $5 \mu \mathrm{g} / \mathrm{ml}$ transferrin and $5 \mathrm{ng} / \mathrm{ml}$ sodium selenite), $0.1 \mathrm{IU} / \mathrm{ml}$ recombinant follicle-stimulating hormone (Serono, Feltham, UK), $0.01 \mathrm{IU} / \mathrm{ml}$ recombinant luteinising hormone (Serono) and $5 \% \mathrm{v} / \mathrm{v}$ foetal bovine serum in $30 \mathrm{~mm}$ Nunc Petri dishes (Scientific Laboratory Supplies, Wilford, UK) and overlaid with washed, equilibrated, embryo-tested mineral oil. Culture was done at $37^{\circ} \mathrm{C}$, in a humidified incubator containing $5 \% \mathrm{CO}_{2}$ in air. On day 0 , follicles were placed in $10 \mu \mathrm{l}$ droplets of follicle culture medium. On day 2 , a further $10 \mu \mathrm{l}$ medium were added. On days 4, $6,8,10$ and $12,10 \mu \mathrm{l}$ culture medium in each droplet were removed and replenished with $10 \mu \mathrm{l}$ fresh medium, using a positive displacement pipette; the spent culture media were stored under oil in Eppendorf tubes at $-80^{\circ} \mathrm{C}$ until analysis. Follicle morphology was recorded during each media change using an inverted microscope: follicles with a spherical morphology with no obvious sign of basement membrane rupture and granulosa proliferation were classed as 'intact'; follicles with evident expansion of the granulosa compartment through a ruptured basement membrane were classed as 'intermediate'; follicles with extensive granulosa proliferation and plating were classed as 'diffuse'; diffuse follicles showing antral-like cavities were classed as 'antral' and follicles which extruded their oocyte or showed morphological signs of apoptosis were classed as 'degenerate'. On day 12, the fresh medium was supplemented such that the final concentration in the droplets was $1.5 \mathrm{IU} / \mathrm{ml}$ for recombinant human chorionic gonadotrophin (hCG; Serono) and $5 \mathrm{ng} / \mathrm{ml}$ for recombinant epidermal growth factor, to induce maturation and ovulation of the OCC. Ovulation in vitro was assessed $16 \mathrm{~h}$ post-hCG and was defined as detachment of an expanded OCC from the follicle. These in vitro-derived OCCs were subsequently harvested for metabolism assessment. 


\section{Measurement of oocyte diameter in preantral follicles}

A subset of oocytes from freshly isolated follicles $100-130 \mu \mathrm{m}$ in diameter was collected to quantify oocyte size and volume at the start of culture. Oocytes were denuded by incubation in $1 \mathrm{mg} / \mathrm{l}$ trypsin in PBS at $37^{\circ} \mathrm{C}$ for $\sim 10 \mathrm{~min}$. The granulosa cells were stripped from the oocyte by repeated aspiration through pipettes of decreasing internal bore.

\section{In vivo-grown OCCs}

OCCs were collected from 6- to 8-week-old mice. Ovarian stimulation was induced by administration of an i.p. dose of $5 \mathrm{IU}$ pregnant mares' serum gonadotrophin (Intervet, Organon, Milton Keynes, UK) followed by $5 \mathrm{IU}$ hCG $46 \mathrm{~h}$ later (Intervet). Oviducts were collected $18 \mathrm{~h}$ post-hCG, and ovulated OCCs were released by puncturing the oviducts with a sterile needle.

\section{Culture of OCCs and stripped oocytes}

OCCs harvested after ovulation in vitro or in vivo were transferred in minimum volume $(<5 \mathrm{nl})$ and cultured singly in $250 \mathrm{nl}$ droplets of modified KSOM (OCCKSOM) containing $1 \mathrm{mM}$ glucose without lactate, glutamine or pyruvate. After $2 \mathrm{~h}, 200 \mathrm{nl}$ samples of spent media were removed and stored under mineral oil at $-80{ }^{\circ} \mathrm{C}$ for future analysis. The OCCs were incubated briefly in $0.5 \mu \mathrm{l}$ hyaluronidase solution $(100 \mathrm{IU} / \mathrm{ml})$ to remove their cumulus cells. Denuded oocytes (DOs) were washed and transferred in a volume of $<0.2-30 \mathrm{nl}$ droplets of modified KSOM (DO-KSOM) containing $0.2 \mathrm{mM}$ sodium pyruvate without lactate or glutamine, for an incubation of $1 \mathrm{~h}$. After incubation, the stage of nuclear maturity was recorded according to stage: germinal vesicle (GV), GV breakdown (GVBD) or metaphase II. Culture plates containing spent media were stored under oil at $-80{ }^{\circ} \mathrm{C}$ until analysis.

\section{Metabolism assays}

Samples of spent follicle culture media were diluted in chromatography-grade water (VWR, Lutterworth, UK) for analysis of nutrient content using a Cobas Mira Automated Analyser (Roche Diagnostics). Samples were diluted 100-, 90- and 20-fold in preparation for duplicate analysis of glucose, lactate and pyruvate concentrations respectively. Oocyte and OCC spent culture media were analysed for metabolite content using the ultramicrofluorescence method described by Leese \& Barton $(1984,1985)$. ATP production from glycolysis was calculated based on the production of two molecules of ATP from one molecule of glucose metabolised by glycolysis.

\section{Statistical analysis}

ANOVA and Kruskal-Wallis with Mann-Whitney $U$ tests were used to analyse differences between culture groups for parametric and non-parametric data respectively. The Wilcoxon signed-rank test was used to assess any differences in the rates of increase in follicle glucose consumption and lactate production before and after addition of hCG. Linear regression analysis was used to assess the relationship between: 1) follicle diameter at the start of culture and follicle/OCC/oocyte metabolic profile; 2) follicle metabolic profile and OCC/oocyte metabolism and 3) OCC metabolism and oocyte pyruvate consumption. Differences were taken to be significant if $P \leq 0.05$.

\section{Results}

\section{Metabolism of in vitro-grown follicles}

Cultured follicles remained relatively intact during the first 4 days of culture (intact classification), but granulosa proliferation and basement membrane rupture became apparent after 4-6 days of culture, forming follicles with a morphology intermediate between intact and diffuse (intermediate classification). Extensive granulosa proliferation caused follicles to assume a diffuse threedimensional morphology (diffuse classification) and formed antral-like cavities by days 8-10 (antral classification). Out of the 517 follicles cultured, 218 (42\%) ovulated OCCs $\sim 15 \mathrm{~h}$ post-hCG.

Follicle metabolism was analysed in 62 of the cultured follicles. Glucose consumption and lactate production rates rose significantly throughout the culture period. Up to day 12, the average rates of increase in glucose consumption and lactate production were approximately linear, but after hCG exposure, there was a significant stimulation $(P<0.0001)$ relative to the trajectories of days $0-12$ (Fig. 1). Follicle morphology influenced glucose and lactate metabolism, with intermediate and diffuse follicles tending to consume more glucose and produce more lactate than follicles that were still intact (Fig. 2). These differences became significant by days $4-6$. On days $6-8$ and $8-10$, antral follicles had significantly greater metabolism of glucose and lactate when compared with diffuse follicles. Interestingly, antral follicle glucose and lactate metabolism did not vary significantly between days 6 and 12, but hCG resulted in highly significant changes, with increases in glucose consumption and lactate production of 2.05- and 1.81-fold respectively (Fig. 2). There were no significant relationships $(P>0.05)$ between initial follicle diameter, degree of theca coverage or oocyte-granulosa apposition at the start of culture and follicle glucose or lactate metabolism. Pyruvate concentrations in spent follicle culture media were consistently below the limit of detection of the assay (threshold $0.025 \mathrm{mM}$ ); therefore, follicle pyruvate metabolism could not be determined. 


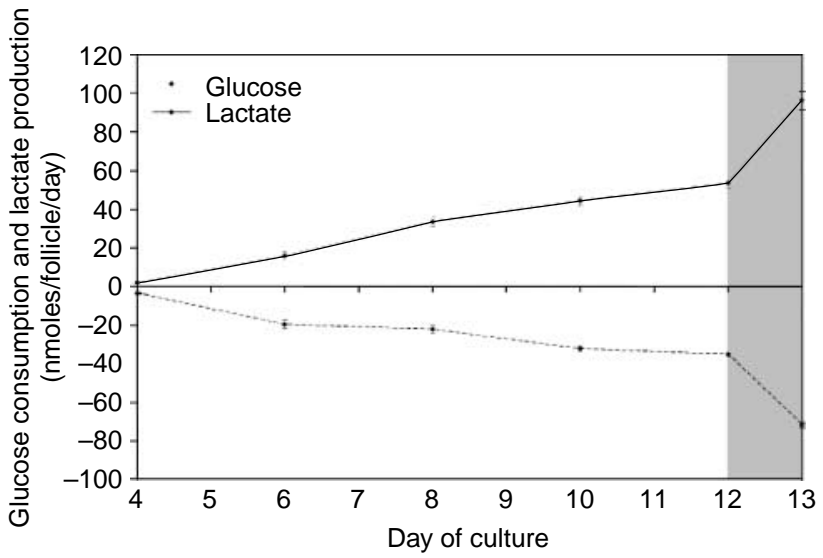

Figure 1 Trajectories of glucose and lactate metabolism rate increases preand post-hCG exposure. Positive values indicate nutrient production and negative values indicate nutrient consumption. Until day 12 , the trajectories of the rates of increase in glucose consumption and lactate production were approximately linear, but after addition of hCG (shaded area), there was a significant stimulation of metabolic activity.

\section{Change in glycolytic index during culture}

The glycolytic index was calculated as the ratio of the number of moles of lactate formed from one mole of glucose. A ratio of 1:2 was assumed to indicate that $100 \%$ of glucose consumed had formed lactate, by oxygenindependent glycolysis. Ratios of $<1: 2$ therefore indicate the proportion of glucose metabolised by oxygenindependent glycolysis but do not account for aerobic glycolysis or Krebs cycle activity. Within each time point, there were no significant shifts in glycolytic index according to follicle morphology (Fig. 3a). During the first 4 days of culture, lactate production accounted for $\sim 24-37 \%$ of glucose consumption, indicating that the minimum proportion of glucose metabolised solely via glycolysis was low. Subsequent time points showed significant increases in glycolytic glucose consumption to a maximum of $60 \%$ between days 6 and 8 . These increases coincided with acquisition of diffuse and antral morphologies. Exposure to hCG caused the proportion of glucose driven solely through glycolysis to decrease significantly to $34 \%$ (Fig. 3a). Calculation of the glycolytic index facilitated determination of glycolytic glucose consumption, from which ATP production from glycolysis was estimated (Fig. 3b). Glycolytic ATP production increased throughout culture with a marked rise in energy production after ovulation induction in vitro (Fig. 3b).

\section{Correlation of follicle metabolism with ovulation rate and OCC metabolism}

Follicle glucose metabolism was not a predictor of ovulation; however, lactate production between days 4-6 and 6-8 was significantly lower in follicles that ovulated in vitro, although there were no significant corresponding differences in glycolytic indices. The rate of increase in (a)
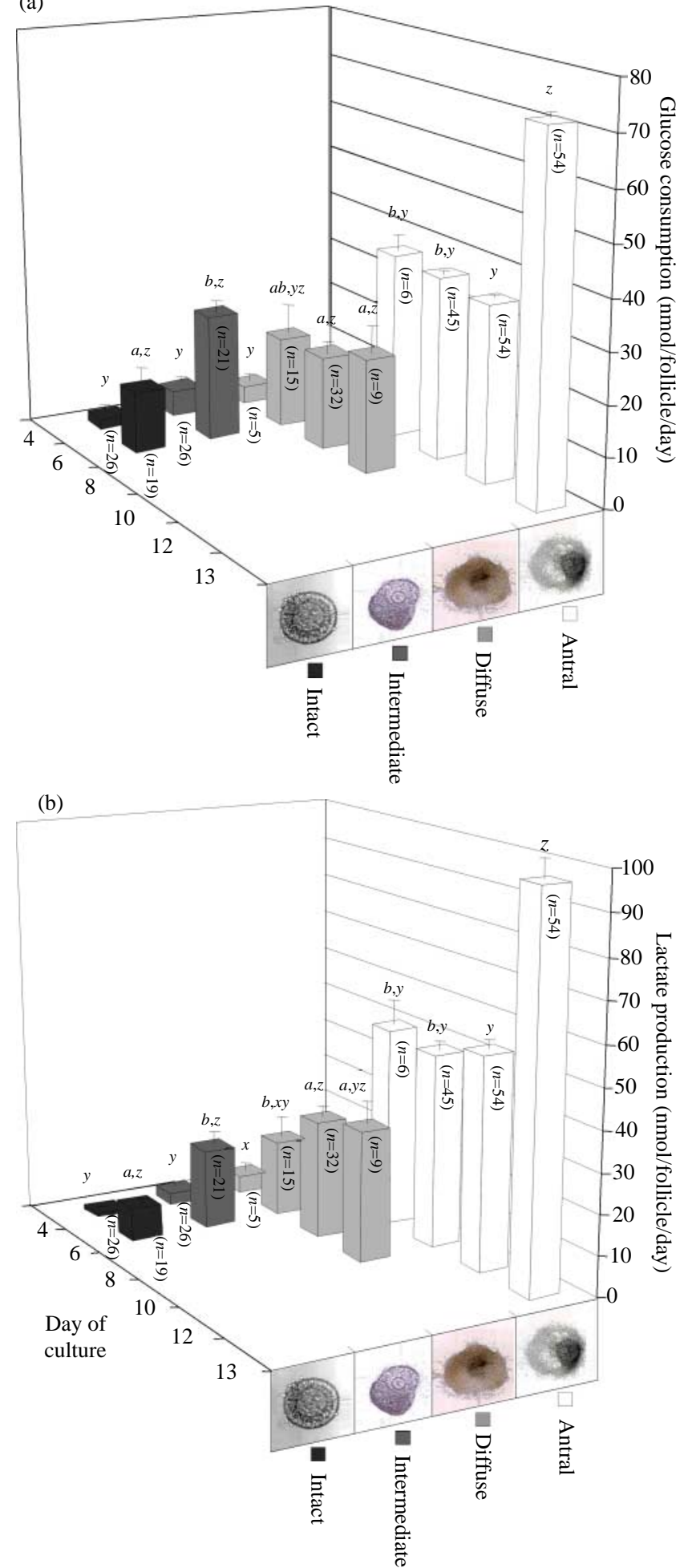

Figure 2 Glucose consumption (a) and lactate production (b) by mouse follicles grown in vitro. Values are means in nmoles per follicle per day \pm S.E.M., with the number of observations shown in parentheses. Within time points, values sharing at least one common letter ( $a$ or $b$ ) are not significantly different $(P>0.05)$. Within morphology groups, values sharing at least one common letter $(x, y$ or $z)$ are not significantly different $(P>0.05)$. 

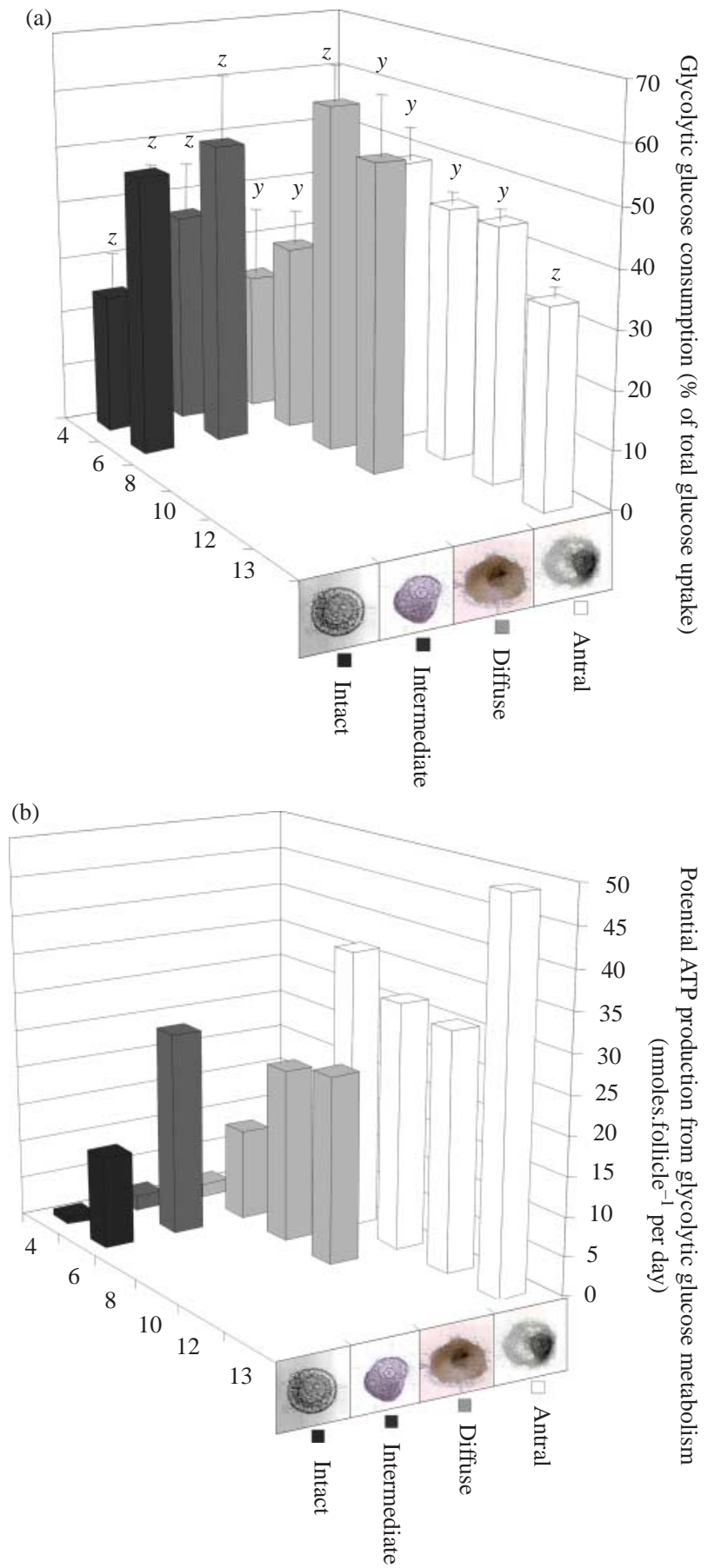

Figure 3 Glycolytic indices (a) and potential ATP generation from glycolytic glucose consumption (b) of mouse follicles grown in vitro. Values are means ( \pm S.E.M). Within the same time points, glycolytic glucose consumption did not differ significantly $(P>0.05)$. For the four morphology groups throughout culture, glycolytic glucose consumption values sharing at least one common letter ( $y$ or $z)$ were not significantly different $(P>0.05)$.

follicle glucose consumption up to day 12 was positively correlated with OCC glucose consumption $(P=0.05)$ and pyruvate production $(P=0.001)$. The rate of increase in follicle lactate production up to day 12 was positively correlated with OCC lactate production $(P=0.025)$.

\section{Oocyte growth during culture}

Oocytes derived from in vitro-grown follicles significantly increased in size during the culture period. At the start of culture, oocytes from preantral follicles had a mean diameter of $57 \pm 0.6 \mu \mathrm{m}(n=97)$ equivalent to a cell volume of $97 \mathrm{pl}$. A significant increase to $69 \pm 2 \mu \mathrm{m}$ in diameter was observed after 13 days of culture, equivalent to a volume of $167 \mathrm{pl}$. The diameter of in vitro-grown oocytes was, however, significantly less than matched controls after ovulation in vivo with a diameter of $71 \pm 3 \mu \mathrm{m}$, equivalent to a cell volume of $230 \mathrm{pl}$.

\section{Comparison of metabolism between in vitro-grown and in vivo-derived control oocytes}

Glucose, lactate and pyruvate metabolism by in vitrogrown and control OCCs were compared following ovulation (Fig. 4). The metabolic patterns of in vitrogrown and control OCCs were similar: glucose was consumed, whereas pyruvate and lactate were produced. The proportions of glucose metabolised to lactate and pyruvate were also almost identical, with ratios close to 1:2 observed for both the in vivo- and in vitrogrown OCCs respectively. However, the absolute rates of nutrient consumption and production by the in vitrogrown OCCs were significantly higher, with each nutrient being metabolised at a rate $\sim 60 \%$ greater than by the in vivo-grown control OCCs (Fig. 4). In addition, pyruvate consumption by in vitro-grown oocytes (1.18 $\pm 0.06 \mathrm{pmol} /$ oocyte per $\mathrm{h}, n=135)$ was significantly lower than by control oocytes $(1.65 \pm 0.06 \mathrm{pmol} /$ oocyte per $\mathrm{h}=208)$. However, when the data were normalised for differences in oocyte volume, pyruvate metabolism was similar per unit cell volume between the two groups $(P>0.05)$.

\section{Factors affecting OCC metabolism}

Lactate production was significantly higher in OCCs harvested from theca grade 2 follicles $(<50 \%$ theca coverage), while lactate production was significantly lower in OCCs harvested from follicles with a poor oocyte-granulosa apposition (connection grade 3 ) at the beginning of culture (Table 1). There were no significant differences in OCC glucose or pyruvate metabolism. Stage of oocyte meiosis significantly influenced glucose consumption and lactate production by in vitro-ovulated OCCs (Fig. 5), with greater metabolism by OCCs containing mature oocytes. Data from OCCs containing immature GV-stage oocytes were too few to analyse. There was no significant difference in pyruvate production.

\section{Factors affecting oocyte pyruvate consumption}

Pyruvate metabolism varied with the stage of nuclear maturation in both in vitro-grown and control oocytes 


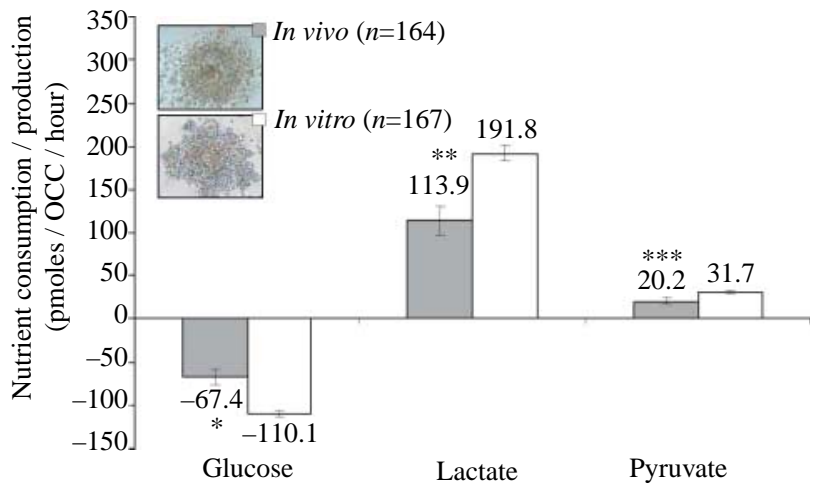

Figure 4 Glucose, lactate and pyruvate metabolism by in vitro- and in vivo-ovulated oocyte-cumulus complexes (OCCs), representative images inset. Values are means \pm s.E.M. for the numbers of follicles shown in parentheses. Positive values indicate nutrient production and negative values indicate nutrient consumption. Statistical comparisons were made for each metabolite: ${ }^{*} P=0.0004,{ }^{* *} P=0.011$ and ${ }^{* * *} P=0.017$.

(Table 2) after hCG exposure, with metaphase II oocytes tending to consume less pyruvate than control germinal vesicle-stage oocytes or in vitro-grown oocytes after germinal vesicle breakdown.

\section{Discussion}

Metabolic profiling provides an overview of nutritional demands occurring within the follicle at different stages with potential implications for developmental competence. In the present study, follicles were cultured separately, and profiling was conducted non-invasively, allowing the metabolism of individual follicles to be followed from preantral to antral stages and enabling data for oocytes to be traced back to their original follicle. Cultured follicles demonstrated an impact of morphology and stage of development on patterns of glucose and lactate metabolism during the 13 days of culture. Low glucose consumption and a low glycolytic index in the early days of culture were followed by an increase in consumption and a rising glycolytic index as development progressed. Metabolic profiling of in vitroand in vivo-ovulated OCCs as well as their oocytes, assessed separately, revealed differences in rates of nutrient turnover, highlighting a potentially important manifestation of long-term culture.

Glucose is a vital nutrient for mouse preantral follicles and was consumed in increasing quantities as follicles developed from the late primary/early secondary stage to ovulation. Likewise, lactate production also increased steadily and significantly through culture. Lactate export is the consequence of non-oxidative (solely glycolytic) glucose metabolism and, at the start of culture, $24-37 \%$ of glucose was metabolised almost exclusively by glycolysis. At this stage, oocytes are surrounded by only one to four layers of cells and are no further than $37 \mu \mathrm{m}$ from the basement membrane, which separates the avascular granulosa cells from the perfused outer layers. This distance is sufficient for maintaining adequate oxygen tension, as predicted in murine and bovine OCCs, which have diameters up to $320 \mu \mathrm{m}$ (Clark et al. 2006). The low level of non-oxidative glucose metabolism in the first few days of culture suggested that glycolysis may not be a major contributor to energy production in preantral follicles until just prior to antrum formation. At this stage, ATP production from glycolysis was $<2 \mathrm{nmol}$ per day. If the oxygen supply is not limiting, glucose can also be metabolised oxidatively, which is far more efficient for energy production. Some glucose may have been diverted down the pentose phosphate pathway, but this accounts for $\leq 1.5 \%$ of total glucose utilisation in murine OCCs (Downs \& Utecht 1999). In any case, up to $76 \%$ of glucose was utilised by cellular processes other than solely by glycolysis. As follicle growth and development progressed, glycolysis accounted for a much greater proportion of glucose consumption, peaking at the start of antrum formation on days 6-8. In consequence, glycolytic ATP production also increased with a marked increase in energy

Table 1 Influence of follicle theca coverage and oocyte-granulosa apposition at the start of culture on metabolism of in vitro-ovulated mouse oocytecumulus complexes.

\begin{tabular}{|c|c|c|c|c|c|c|}
\hline & \multicolumn{2}{|c|}{ Theca coverage } & \multicolumn{4}{|c|}{ Oocyte-granulosa apposition } \\
\hline & $\begin{array}{l}\text { Grade } 1 ; \\
>50 \% \\
\text { coverage } \\
(n=111)\end{array}$ & $\begin{array}{l}\text { Grade } 2 ; \\
<50 \% \\
\text { coverage } \\
(n=15)\end{array}$ & $\begin{array}{l}\text { Grade } 1 ; \\
>2 / 3 \\
\text { good } \\
\text { apposition } \\
(n=68)\end{array}$ & $\begin{array}{l}\text { Grade 2; } \\
1 / 3-2 / 3 \\
\text { good } \\
\text { apposition } \\
(n=18)\end{array}$ & $\begin{array}{l}\text { Grade } 3 ; \\
<1 / 3 \text { good } \\
\text { apposition } \\
(n=42)\end{array}$ & Exise \\
\hline $\begin{array}{l}\text { OCC glucose } \\
\text { consumption }\end{array}$ & $109.1 \pm 4.7$ & $122.2 \pm 10$ & $114.7 \pm 5.7$ & $93.0 \pm 12.4$ & \multicolumn{2}{|c|}{$111.8 \pm 7.5$} \\
\hline $\begin{array}{l}\text { OCC lactate } \\
\text { production }\end{array}$ & $163 \pm 12^{a}$ & $254 \pm 21^{b}$ & $200 \pm 14^{y}$ & $203 \pm 32^{y}$ & \multicolumn{2}{|c|}{$128 \pm 18^{z}$} \\
\hline $\begin{array}{l}\text { OCC pyruvate } \\
\text { production }\end{array}$ & $37.6 \pm 3.7$ & $34.5 \pm 3.7$ & $35.5 \pm 3.4$ & $35.9 \pm 9.3$ & \multicolumn{2}{|c|}{$40.6 \pm 7.3$} \\
\hline
\end{tabular}

Values are means (pmol/OCC per h) \pm s.E.M. for the numbers of OCCs $(n)$ shown in parentheses. Absent or identical letters indicate no significance $(P>0.01)$. Comparisons are confined to each metabolite within each classification. 


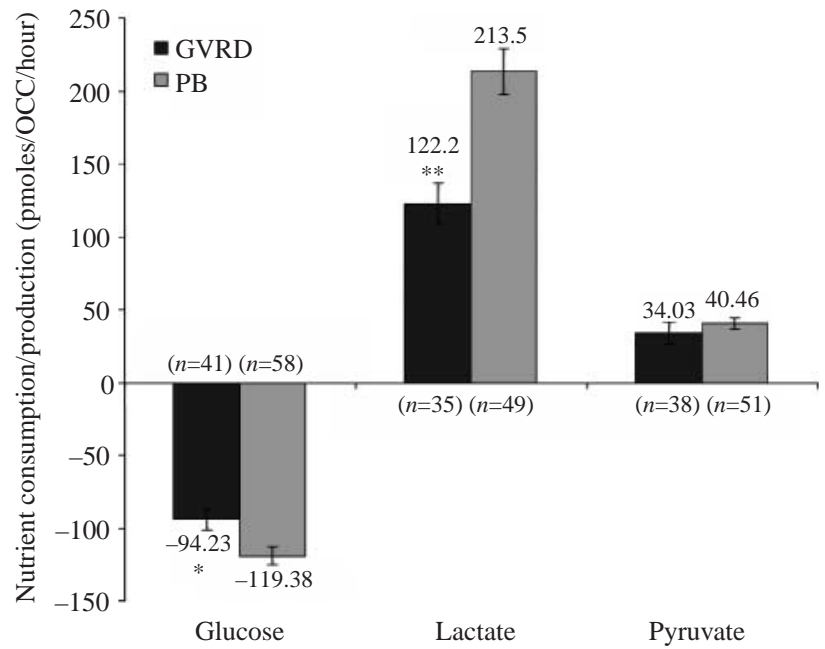

Figure 5 Glucose, lactate and pyruvate metabolism by in vitro-ovulated oocyte-cumulus complexes containing GVBD or metaphase II (PB) oocytes. Values are means \pm s.E.M. of the number of OCCs shown in parentheses. Negative values indicate nutrient consumption and positive values indicate nutrient production. Statistical comparisons were made for each metabolite: ${ }^{*} P=0.009$ and ${ }^{* *} P=0.000$.

production after ovulation induction in vitro. Several energy-demanding processes requiring ATPare known to be triggered by ovulation induction, such as progesterone production (Peluso 2006) and luteal cell differentiation (Boos et al. 1988). Glucose is also necessary for cumulus expansion (Sutton-McDowall et al. 2004) and purine synthesis (Downs et al. 1998).

The predominance of glycolytic energy production in large follicles is well documented (Boland et al. 1993, Gull et al. 1999), and the enzymatic machinery for oxidative glucose metabolism is already present at preantral stages, including human follicles (Roy \& Terada 1999). It is unlikely that glycolysis is the sole source of energy in mouse follicles, because inhibition of aerobic metabolism seriously inhibits normal growth (Wycherley et al. 2005). However, fully grown, but not growing, mouse oocytes promote the expression of glycolytic pathway components in cumulus cells (Sugiura et al. 2005), a phenomenon that is consistent with our observations of glycolysis only accounting for a small fraction of glucose used by preantral follicles, which contain small oocytes. In follicles with incipient antrum formation, and fullsized oocytes, on the other hand, a peak in glycolytic glucose utilisation was seen.

Changes in metabolism were also associated with changes in follicle morphology. This was particularly evident in larger antral follicles that had significantly greater glucose consumption and lactate production rates than those follicles that, after the same time in culture, remained 'diffuse'. It has recently been demonstrated that several genes associated with glycolysis are upregulated in cumulus when compared with mural granulosa cells, with the levels of expression related to the distance from the oocyte (Sugiura et al. 2005).

Ovulation induction elicited large rises in the rates of glucose consumption and lactate production (2.0- and 1.8 -fold respectively), a mechanism that could be explained by upregulation of hexokinase activity (Downs et al. 1998). During ovulation induction in vitro, the actual proportion of glucose metabolised glycolytically decreased significantly and, in conjunction with a major increase in glucose consumption, this indicates that a substantial portion of glucose was being metabolised by pathways other than glycolysis. Since oestradiol secretion decreases prior to ovulation (Spears et al. 1998), this effect could help to account for reduced oxygen requirements, thus benefiting the supply for oxidative energy production. It is also highly possible that a greater proportion of glucose was diverted through the pentose phosphate pathway to support the resumption of meiosis (Downs et al. 1998).

Future studies may investigate the influence of culture media composition and energy metabolite concentrations on metabolism of cultured follicles. In oocytes and embryos, culture medium composition is well known to adjust rates of metabolism and development (Conaghan et al. 1998, Roberts et al. 2002), and nutrient ratio can also be influential (Chatot et al. 1989, Lane \& Gardner 2000). It is likely that cultured follicles will also be susceptible to the culture environment, for example, glucose metabolism by cultured preovulatory mouse follicles exposed to glucose concentrations of 1-2 mM has glucose consumption rates approximately fourfold lower (Boland et al. 1994b) than those observed in the current study, in which $5.5 \mathrm{mM}$ glucose was used. Oxygen availability can also profoundly affect follicle

Table 2 Pyruvate consumption by mouse in vitro- and in vivo-ovulated oocytes at different stages of nuclear maturation.

\begin{tabular}{lll}
\hline Oocyte stage & $\begin{array}{l}\text { In vitro-ovulated oocytes } \\
\text { (pmol/oocyte per } \mathrm{h})\end{array}$ & \\
\hline Germinal vesicle intact & $1.54 \pm 0.31(n=3)^{a b, x}$ & $2.25 \pm 0.10(n=6)^{a, x}$ \\
Germinal vesicle breakdown & $1.30 \pm 0.10(n=52)^{a, x}$ & $1.99 \pm 0.07(n=5)^{a b, y}$ \\
Metaphase II & $1.13 \pm 0.06(n=66)^{b, x}$ & $1.49 \pm 0.06(n=101)^{b, y}$
\end{tabular}

In columns, values with at least one common letter $(a$ and $b)$ are not significantly different, $P>0.05$. In rows, values with at least one common letter $(x$ and $y$ ) are not significantly different, $P>0.05$ ). 
metabolism (Wycherley et al. 2005). All this highlights the requirement for further thorough investigation into factors influencing metabolism of cultured follicles and characterisation of the endocrine and nutritional milieu to which they are exposed naturally in vivo.

Metabolic profiling of in vitro-grown oocytes and comparison with their in vivo-ovulated counterparts provide valuable information about the influence of the culture environment on oocyte biology. Oocytes grown in vitro are fertilisable (Cortvrindt et al. 1996, Rose et al. 1999), but ultimately, their developmental competence is lower than in vivo-derived oocytes. Oogenesis requires a complex and timely sequence of highly coordinated biological events. Data from other longterm murine follicle culture studies (Eppig et al. 1996, Eppig \& O'Brien 1998, Obata et al. 2002, O'Brien et al. 2003) consistently show reduced maturation and fertilisation rates, implying that key biological processes are altered during prolonged exposure to culture conditions. The present study has revealed that these culture-induced changes include altered metabolic profiles, although whether this is due to altered responsiveness to endocrine stimuli or post-transduction lesions or differences in cumulus cell numbers is unclear. It is also possible that the difference in age between the mice used for harvesting preantral follicles for in vitro growth and those used for superovulation may affect metabolism, although this is unlikely, as qualitative differences observed between follicles from immature and adult rats appear to be due to endocrine differences, and inevitably, there are large endocrine differences between conditions in vivo and in vitro. Increased nutrient metabolism may be deleterious to oocyte developmental competence, as excessive lactate output is associated with cellular stress (Elekes et al. 1996) and may occur as a result of the upregulation of glycolytic metabolism and downregulation of oxidative energy production to reduce production of reactive oxygen species. Despite differences in metabolism rates between in vitro-ovulated and control OCCs, the ratios of glucose consumption to lactate and pyruvate production were very similar between in vivo- and in vitro-ovulated OCCs, both with ratios of almost 1:2, indicating that all the glucose consumed by OCCs could be accounted for by lactate and pyruvate secretion.

Oocyte pyruvate consumption was lower in cultured oocytes, but when oocyte volume was taken into account, it was similar in the two groups. Since pyruvate is metabolised aerobically, the data imply that mitochondrial density or activity is similar per unit volume of oocyte between in vitro-grown and normal oocytes. In vitro-grown oocytes had a significantly smaller volume, again highlighting suboptimal conditions, and smaller oocytes have previously been observed in studies of cultured mouse follicles (Cortvrindt et al. 1996, Eppig et al. 1996).
Oocytes at metaphase I consumed significantly more pyruvate than those at metaphase II. This corroborates previous findings in mouse (Downs et al. 2002) and bovine oocytes (Rieger \& Loskutoff 1994, Gandolfi et al. 1998, Steeves et al. 1999), which showed elevated consumption of oxidisable substrates during maturation. This phenomenon is thought to satisfy the greater energy requirement during germinal vesicle breakdown, spindle formation and chromosome segregation. The stage of oocyte meiosis also influenced glucose and lactate metabolism of OCCs, with greater glucose and lactate metabolism by OCCs containing a metaphase II oocyte. The causative factor for this is unclear, but may involve meiosis-induced alterations to oocyte-cumulus metabolic cooperativity, as is the case in mouse OCCs, containing an oocyte held in meiotic arrest, which consume more glucose than OCCs containing an actively maturing oocyte (Downs et al. 2002).

Metabolic analysis of OCCs derived from follicles tracked through culture can be informative about the quality of the original follicle. Follicles with fewer theca cells produced OCCs with a higher lactate formation, an indicator of cellular stress (Elekes et al. 1996). It is possible that the theca-enclosed follicles used in the present work produced better quality OCCs, as theca cells influence the developmental competence of bovine OCCs (Kotsuji et al. 1994, Richard \& Sirard 1996). During growth, the oocyte is linked via gap junctions to granulosa cells, forming a bidirectional communication pathway, which is critical for normal development (Albertini et al. 2001, Eppig et al. 2002, 2005, Sugiura et al. 2005). In mice with defective follicle gap junctions, oocytes fail to complete development (Simon et al. 1997, Ackert et al. 2001). In this study, cultured follicles with impaired granulosa-oocyte apposition were less likely to ovulate in vitro, and those that succeeded produced OCCs with reduced glycolytic glucose utilisation. In this situation, reduced oocyte-follicle coupling may promote premature luteinisation (el-Fouly et al. 1970). In support of this idea, oocytectomised murine OCCs show reduced expression of glycolysis components and, during bovine luteal development, activities of key pentose phosphate pathway and Krebs cycle enzymes increase (Boos et al. 1988), implying that a greater proportion of glucose is metabolised through these pathways, reducing lactate output.

Mouse follicles have been cultured from the late primary stage to maturity and their metabolic profiles during this period have been characterised. During preantral development, follicles utilise a combination of glycolysis and aerobic glucose metabolism but during antrum formation glucose consumption is predominantly glycolysis driven. The metabolism of OCCs and metaphase II denuded oocytes harvested from in vitrogrown ovulated follicles differed significantly from those ovulated in vivo. Furthermore, OCC nutrient metabolism dynamics were altered by theca cells and 
oocyte-granulosa apposition, implying that the presence of all follicle cell types and sufficient crosstalk between the germ cell and somatic compartments are critical factors for oogenesis and folliculogenesis. Study of the metabolic processes within the whole OCC and not just the isolated oocyte promotes a greater understanding of the roles of metabolism and nutrient availability in promoting oocyte developmental competence.

\section{Acknowledgements}

The authors are grateful to Dr Rita Cortvrindt for expert advice regarding mouse follicle culture and to Mr Peter Humpherson (University of York, UK) for assistance during use of the Cobas Mira. This work was supported by a Universities of Leeds \& York White Rose Scholarship and a grant from Candlelighters. The authors declare that there is no conflict of interest that would prejudice the impartiality of this scientific work.

\section{References}

Ackert CL, Gittens JE, O'Brien MJ, Eppig JJ \& Kidder GM 2001 Intercellular communication via connexin43 gap junctions is required for ovarian folliculogenesis in the mouse. Developmental Biology 233 258-270.

Albertini DF, Combelles CM, Benecchi E \& Carabatsos MJ 2001 Cellular basis for paracrine regulation of ovarian follicle development. Reproduction 121 647-653.

Biggers J, Whittingham D \& Donahue R 1967 The pattern of energy metabolism in the mouse oocyte and zygote. PNAS 58567.

van Blerkom J 1998 Epigenetic influences on oocyte developmental competence: perifollicular vascularity and intrafollicular oxygen. Journal of Assisted Reproduction and Genetics 15 226-234.

Boland NI, Humpherson PG, Leese HJ \& Gosden RG 1993 Pattern of lactate production and steroidogenesis during growth and maturation of mouse ovarian follicles in vitro. Biology of Reproduction $\mathbf{4 8}$ 798-806.

Boland NI, Humpherson PG, Leese HJ \& Gosden RG 1994a Characterization of follicular energy metabolism. Human Reproduction $\mathbf{9}$ 604-609.

Boland NI, Humpherson PG, Leese HJ \& Gosden RG 1994b The effect of glucose metabolism on murine follicle development and steroidogenesis in vitro. Human Reproduction 9 617-623.

Boos A, Meyer W, Peukert-Adam I, Schwarz R \& Grunert E 1988 Delta 5-3 $\beta$-hydroxysteroid dehydrogenase, succinate dehydrogenase and glucose-6-phosphate dehydrogenase in the bovine corpus luteum of estrous cycle: a quantitative histochemical study. Gegenbaurs Morphologisches Jahrbuch 134 189-204.

Calder MD, Caveney AN, Sirard MA \& Watson AJ 2005 Effect of serum and cumulus cell expansion on marker gene transcripts in bovine cumulus-oocyte complexes during maturation in vitro. Fertility and Sterility 83 1077-1085.

Chatot CL, Ziomek CA, Bavister BD, Lewis JL \& Torres I 1989 An improved culture medium supports development of random-bred 1 -cell mouse embryos in vitro. Journal of Reproduction and Fertility 86 679-688.

Clark AR, Stokes YM, Lane M \& Thompson JG 2006 Mathematical modelling of oxygen concentration in bovine and murine cumulusoocyte complexes. Reproduction 131 999-1006.

Conaghan J, Hardy K, Leese HJ, Winston RM \& Handyside AH 1998 Culture of human preimplantation embryos to the blastocyst stage: a comparison of 3 media. International Journal of Developmental Biology 42 885-893.
Cortvrindt R, Smitz J \& Van Steirteghem AC 1996 In vitro maturation, fertilization and embryo development of immature oocytes from early preantral follicles from prepuberal mice in a simplified culture system. Human Reproduction 11 2656-2666.

Downs S \& Utecht A 1999 Metabolism of radiolabeled glucose by mouse oocytes and oocyte-cumulus cell complexes. Biology of Reproduction 60 1446-1452.

Downs SM, Humpherson PG \& Leese HJ 1998 Meiotic induction in cumulus cell-enclosed mouse oocytes: involvement of the pentose phosphate pathway. Biology of Reproduction 58 1084-1094.

Downs SM, Humpherson PG \& Leese HJ 2002 Pyruvate utilization by mouse oocytes is influenced by meiotic status and the cumulus oophorus. Molecular Reproduction and Development 62 113-123.

Elekes O, Venema K, Postema F, Dringen R, Hamprecht B \& Korf J 1996 Evidence that stress activates glial lactate formation in vivo assessed with rat hippocampus lactography. Neuroscience Letters 208 69-72.

Eppig J \& O'Brien M 1998 Comparison of preimplantation developmental competence after mouse oocyte growth and development in vitro and in vivo. Theriogenology 49 415-422.

Eppig JJ, O'Brien M \& Wigglesworth K 1996 Mammalian oocyte growth and development in vitro. Molecular Reproduction and Development 44 260-273.

Eppig J, Wigglesworth K \& Pendola FL 2002 The mammalian oocyte orchestrates the rate of ovarian follicular development. PNAS 99 2890-2894.

Eppig JJ, Pendola FL, Wigglesworth K \& Pendola JK 2005 Mouse oocytes regulate metabolic cooperativity between granulosa cells and oocytes: amino acid transport. Biology of Reproduction $\mathbf{7 3}$ 351-357.

Fernandez-Gonzalez R, Moreira P, Bilbao A, Jimenez A, PerezCrespo M, Ramirez MA, Rodriguez De Fonseca F, Pintado B \& Gutierrez-Adan A 2004 Long-term effect of in vitro culture of mouse embryos with serum on mRNA expression of imprinting genes, development, and behavior. PNAS 101 5880-5885.

el-Fouly MA, Cook B, Nekola M \& Nalbandov AV 1970 Role of the ovum in follicular luteinization. Endocrinology 87 286-293.

Gandolfi F, Milanesi E, Pocar P, Luciano AM, Brevini TA, Acocella F, Lauria A \& Armstrong DT 1998 Comparative analysis of calf and cow oocytes during in vitro maturation. Molecular Reproduction and Development 49 168-175.

Gosden RG \& Byatt-Smith JG 1986 Oxygen concentration gradient across the ovarian follicular epithelium: model, predictions and implications. Human Reproduction 1 65-68.

Gull I, Geva E, Lerner-Geva L, Lessing JB, Wolman I \& Amit A 1999 Anaerobic glycolysis. The metabolism of the preovulatory human oocyte. European Journal of Obstetrics, Gynecology and Reproductive Biology 85 225-228.

Harris S 2002 Experimental and clinical investigation into mammalian oocyte metabolism, nutrition and fertility. PhD thesis, University of Leeds.

Kotsuji F, Kubo M \& Tominaga T 1994 Effect of interactions between granulosa and thecal cells on meiotic arrest in bovine oocytes. Journal of Reproduction and Fertility 100 151-156.

Lane M \& Gardner DK 2000 Lactate regulates pyruvate uptake and metabolism in the preimplantation mouse embryo. Biology of Reproduction 62 16-22.

Leese HJ \& Barton AM 1984 Pyruvate and glucose uptake by mouse ova and preimplantation embryos. Journal of Reproduction and Fertility 72 9-13.

Leese HJ \& Barton AM 1985 Production of pyruvate by isolated mouse cumulus cells. Journal of Experimental Zoology 234 231-236.

Leese HJ \& Lenton EA 1990 Glucose and lactate in human follicular fluid: concentrations and interrelationships. Human Reproduction $\mathbf{5}$ 915-919.

Nayudu PL \& Osborn SM 1992 Factors influencing the rate of preantral and antral growth of mouse ovarian follicles in vitro. Journal of Reproduction and Fertility 95 349-362. 
Newton H, Picton H \& Gosden RG 1999 In vitro growth of oocytegranulosa cell complexes isolated from cryopreserved ovine tissue. Journal of Reproduction and Fertility 115 141-150.

Obata Y, Kono T \& Hatada I 2002 Oogenesis: maturation of mouse fetal germ cells in vitro. Nature 418 497-498.

O'Brien MJ, Pendola JK \& Eppig JJ 2003 A revised protocol for in vitro development of mouse oocytes from primordial follicles dramatically improves their developmental competence. Biology of Reproduction 68 1682-1686.

Peluso JJ 2006 Multiplicity of progesterone's actions and receptors in the mammalian ovary. Biology of Reproduction 75 2-8.

Picton HM \& Gosden RG 2000 In vitro growth of human primordial follicles from frozen-banked ovarian tissue. Molecular and Cellular Endocrinology 166 27-35.

Picton HM, Danfour MA, Harris SE, Chambers EL \& Huntriss J 2003 Growth and maturation of oocytes in vitro. Reproduction $\mathbf{6 1}$ 445-462.

Richard FJ \& Sirard MA 1996 Effects of follicular cells on oocyte maturation II: theca cell inhibition of bovine oocyte maturation in vitro. Biology of Reproduction 54 22-28.

Rieger D \& Loskutoff N 1994 Changes in the metabolism of glucose, pyruvate, glutamine and glycine during maturation of cattle oocytes in vitro. Journal of Reproduction and Fertility 100 257-262.

Roberts R, Franks S \& Hardy K 2002 Culture environment modulates maturation and metabolism of human oocytes. Human Reproduction 17 2950-2956.

Rose UM, Hanssen RG \& Kloosterboer HJ 1999 Development and characterization of an in vitro ovulation model using mouse ovarian follicles. Biology of Reproduction 61 503-511.

Roy SK \& Terada DM 1999 Activities of glucose metabolic enzymes in human preantral follicles: in vitro modulation by follicle-stimulating hormone, luteinizing hormone, epidermal growth factor, insulin-like growth factor I, and transforming growth factor $\beta 1$. Biology of Reproduction 60 763-768.
Roy SK \& Treacy BJ 1993 Isolation and long-term culture of human preantral follicles. Fertility and Sterility 59 783-790.

Simon AM, Goodenough DA, Li E \& Paul DL 1997 Female infertility in mice lacking connexin 37. Nature 385 525-529.

Spears N, Murray AA, Allison V, Boland NI \& Gosden RG 1998 Role of gonadotrophins and ovarian steroids in the development of mouse follicles in vitro. Journal of Reproduction and Fertility 113 $19-26$.

Steeves TE, Gardner DK, Zuelke KA, Squires TS \& Fry RC 1999 In vitro development and nutrient uptake by embryos derived from oocytes of pre-pubertal and adult cows. Molecular Reproduction and Development 54 49-56.

Sugiura K, Pendola FL \& Eppig JJ 2005 Oocyte control of metabolic cooperativity between oocytes and companion granulosa cells: energy metabolism. Developmental Biology 279 20-30.

Sutton-McDowall ML, Gilchrist RB \& Thompson JG 2004 Cumulus expansion and glucose utilisation by bovine cumulus-oocyte complexes during in vitro maturation: the influence of glucosamine and follicle-stimulating hormone. Reproduction $\mathbf{1 2 8}$ 313-319.

Wycherley G, Kane MT \& Hynes AC 2005 Oxidative phosphorylation and the tricarboxylic acid cycle are essential for normal development of mouse ovarian follicles. Human Reproduction 20 2757-2763.

Received 5 February 2007

First decision 15 March 2007

Revised manuscript received 21 April 2007

Accepted 18 June 2007 